\title{
The Calculation of Fourier Coefficients by the Möbius Inversion of the Poisson Summation Formula. Part III. Functions Having Algebraic Singularities*
}

\author{
By J. N. Lyness
}

\begin{abstract}
The purpose of this paper is to extend the MIPS theory described in Parts I and II to functions having algebraic singularities. As in the simpler cases, the theory is based on expressing the remainder term in the appropriate Fourier coefficient asymptotic expansion as an infinite series, each element of which is a remainder in the Euler-Maclaurin summation formula. In this way, an expression is found for $\int_{a}^{b} f(x) \cos 2 \pi m x d x(0 \leqq$ $a<b \leqq 1$ ) where $f(x)=(x-a)^{\alpha}(b-x)^{\beta} \phi(x), \phi(x)$ being analytic and $\alpha, \beta>-1$. This expression, and variants of it form a convenient basis for the numerical calculation of a set of Fourier coefficients. The calculation requires approximate values of the first few derivatives of $\phi(x)$ at $x=a$ and at $x=b$, together with trapezoidal rule sums over [0,1] of $f(x)$. Some of the incidental constants are values of the generalized zeta function $\zeta(s, a)$.
\end{abstract}

1. Outline of Problem. In Parts I and II (Lyness [1], [2]) of this series, the author has introduced representations for the Fourier coefficients

$$
C^{(m)} f=\int_{0}^{1} f(x) \cos 2 \pi m x d x ; \quad S^{(m)} f=\int_{0}^{1} f(x) \sin 2 \pi m x d x
$$

for various classes of functions $f(x)$. These representations may be applied in the problem of calculating a set of Fourier coefficients to a uniform accuracy. This method of calculation is termed the MIPS method.

In Part I, attention was restricted to functions satisfying

$$
f(x) \in C^{(p)}[0,1]
$$

In the first half of Part II, piecewise continuous functions were considered. This problem was immediately reduced to that of considering $f(x)$ defined by

$$
\begin{array}{ll}
f(x)=\phi(x), & 0<x<b, \\
f(x)=\frac{1}{2} \phi(x), & x=a, x=b, \\
f(x)=0, & x<a, x>b,
\end{array}
$$

where $0<a<b<1$ and

$$
\phi(x) \in C^{(p)}[a, b] .
$$

In the second half of Part II, a special treatment for analytic functions having known poles in the complex plane near the middle of the integration interval was given.

Received December 28, 1970.

AMS 1969 subject classifications. Primary 4210, 6555; Secondary 4208.

Key words and phrases. Fourier coefficients, Euler-Maclaurin summation formula, Fourier coefficient asymptotic expansion, trigonometric integration, numerical quadrature, ignoring the singularity.

* Work performed under the auspices of the United States Atomic Energy Commission. 
In this part (Part III), we conclude this series by generalizing the treatment to functions $f(x)$ which have a finite number of algebraic singularities located on the interval of integration, but which are otherwise analytic on this interval. It suffices to treat only the interval between two adjacent singularities. Thus, we define

$$
f(x)=(x-a)^{\alpha}(b-x)^{\beta} \phi(x), \quad \alpha, \beta>-1,
$$

where

$$
\phi(x) \in C^{(p)}[a, b]
$$

and

$$
f(x)=0, \quad x<a, x>b .
$$

In general, at least one of $\alpha$ or $\beta$ is nonintegral. If both are integers, the theory reduces to that described in Part II, while if in addition $a=0$ and $b=1$, it reduces to that described in Part I.

The theory described in Parts I and II is valid for continuous functions $f(x)$ (or $\phi(x))$ whose first $p$ derivatives are continuous. However, the computational methods based on this theory become successively less useful, the lower the value of $p$. When $f(x)$ has a low value of $p$ by reason of an algebraic singularity of known form, the use of the methods described in this paper is significantly more efficient than those of the previous parts.

The formulas derived in all cases involve trapezoidal rule sums of the form

$$
\begin{aligned}
& R^{[m, 1]} f=\frac{1}{m} \sum_{i=0}^{m} \prime \prime f(j / m), \\
& R^{[m, \nu]} f=\frac{1}{m} \sum_{i=1}^{m} f\left(\frac{j-1+t_{\nu}}{m}\right), \quad t_{\nu}=(1+\nu) / 2, \quad|\nu|<1,
\end{aligned}
$$

and in applications, these sums have to be calculated for $m=1,2, \cdots, \bar{s}$, the terminal value depending on the required accuracy. In addition, approximations for the derivatives $\phi^{(a)}(b), \phi^{(a)}(a), q=1,2, \cdots, p$, are required. However, as in the previous cases, the accuracy of the result need not be affected by the use of only poor approximations for the derivatives.

The formulas derived here are closely related to those considered previously. One difference is that the derivatives which occur in the formulas are $g^{(a)}(a), h^{(a)}(b)$ where

$$
\begin{aligned}
& g(x)=(b-x)^{\beta} \phi(x), \\
& h(x)=(x-a)^{\alpha} \phi(x)
\end{aligned}
$$

and so a subsidiary calculation may be necessary to obtain these from $\phi^{(a)}(a)$ and $\phi^{(a)}(b)$. Another difference is that the incidental coefficients are no longer trivial to compute. For example, the coefficients required in one version of the MIPS method are

$$
\begin{array}{lll}
\bar{\zeta}(-(q+\beta), b m) ; & q=0,1, \cdots, p_{b}-1 ; & m=1,2, \cdots, \bar{s}, \\
\bar{\zeta}(-(q+\alpha),-a m) ; & q=0,1, \cdots, p_{a}-1 ; \quad m=1,2, \cdots, \bar{s} .
\end{array}
$$


Typically, $p_{a}=p_{b}=5$ and $\bar{s}=20$. Here $\bar{\zeta}(s, x)$ is a function of period 1 in the $x$ variable which coincides with the generalized zeta function $\zeta(s, x)$ in the interval $0<x<1$. It is relatively straightforward to write an algorithm to calculate these, but this is of course an inconvenience which does not occur in the special cases treated in Parts I and II. This is because when $q$ is an integer, $\bar{\zeta}(-q, x)$ is related to the periodic Bernoulli function $\bar{B}_{p}(x)$ through the relation

$$
\bar{\zeta}(-q, x)=-\bar{B}_{1+q}(x) /(1+q) .
$$

In Part I, in fact, the only values of $x$ required are $\frac{1}{4}, \frac{1}{2}, \frac{3}{4}$ and 1 and so the only coefficients needed are Bernoulli and Euler numbers.

In the less sophisticated cases dealt with in Parts I and II, the same basic approach was followed as will be followed here. This consists in writing down an asymptotic expansion (the FCAE) for the Fourier coefficient. This asymptotic expansion is then used to derive an asymptotic expansion for the error functional corresponding to a trapezoidal rule, which is a variant of the conventional Euler-Maclaurin expansion. This manipulation is carried out by means of the Poisson summation formula which also yields an expression for the remainder term for the Euler-Maclaurin expansion in terms of remainder terms of the FCAE. The Möbius inversion of this set of equations produces the formula of interest by providing an expression (in the form of an infinite sum) for the remainder term in the FCAE.

In the case under consideration here, the steps in the derivation are identical to those in the previous cases. The difference is that the results are more complicated. Consequently, in the derivation given here the discussion is kept to a minimum. Only points which do not arise in Parts I and II are discussed in any detail.

2. The Fourier Coefficient and Euler-Maclaurin Asymptotic Expansions. The FCAE for the Fourier coefficients (1.1) of the function defined by (1.5) has been derived by Erdélyi [3]. A simpler proof, valid when $\phi(x)$ is analytic in the interval $[a, b]$, is given in Lyness [4]. In terms of the related functions $g(x)$ and $h(x)$ given by (1.10) and (1.11), this FCAE takes the form:

$$
\begin{aligned}
C^{(m)} f+i S^{(m)} f= & \int_{a}^{b} f(x) e^{2 \pi i m x} d x \\
= & -e^{2 \pi i m b-i \pi \beta / 2} \sum_{a=0}^{p_{b}-1} \frac{h^{(a)}(b) i^{q+1}(q+\beta) !}{q !(2 \pi m)^{a+\beta+1}} \\
& +e^{2 \pi i m a+i \pi \alpha / 2} \sum_{a=0}^{p_{a}-1} \frac{g^{(a)}(a) i^{a+1}(q+\alpha) !}{q !(2 \pi m)^{a+\alpha+1}}+C_{p_{b}, p_{a}}^{(m)} f+i S_{p_{b}, p_{a}}^{(m)} f,
\end{aligned}
$$

where the remainder term satisfies the order relation

$$
C_{p_{b}, p_{a}}^{(m)} f+i S_{p_{b}, p_{a}}^{(m)} f \sim O\left(m^{-\min \left(p_{b}+\beta+1, p_{a}+\alpha+1\right)}\right) \text { as } m \rightarrow \infty .
$$

The Poisson summation formula (I, 2.12) may be written

$$
R^{[m, \nu]} f-I f=2 \sum_{r=1}^{\infty} \operatorname{Re}\left[e^{-2 \pi i r t,} \int_{0}^{1} f(x) e^{2 \pi i r m x} d x\right] .
$$

The proof of this formula, given in Part I, Section 2, depends on the circumstance 
that the function coincides with its Fourier series at each point at which function values may be required. Thus:

$$
f\left(x_{i}\right)=\bar{f}\left(x_{i}\right)=I f+2 \sum_{r=1}^{\infty} C^{(r)} f \cos 2 \pi r x_{i}+2 \sum_{r=1}^{\infty} S^{(r)} f \sin 2 \pi r x_{i} .
$$

When $f(x)$ has an infinite singularity at $a$ (or at $b$ ), relation (2.4) is not valid when $x_{i}=a$ (or $b$ ). If the rule sum $R^{[m, \nu]} f$ then involves a function evaluation at $x=a$ (or $b$ ), the derivation of (2.3) fails. In this case, the left-hand side of (2.3) is indeterminate because of the term $f(a)$ (or $f(b)$ ) while the series on the right fails to converge. Consequently, (2.3) is valid for all $\alpha>-1, \beta>-1$ under the following restriction.

Restriction 2.5 .

$$
\begin{aligned}
& \text { For } \alpha<0, \quad m a-t_{\nu} \neq \text { integer, } \\
& \text { for } \beta<0, \quad m b-t_{\nu} \neq \text { integer. }
\end{aligned}
$$

We now proceed to substitute expression (2.1) into the right-hand side of (2.3). The sum over index $r$ may be expressed in terms of the generalized zeta function $\zeta(s, a)$. In particular, we require the sum of the Fourier series

$$
\begin{aligned}
& \bar{\zeta}(-q, x)=2 q ! \sum_{r=1}^{\infty} \frac{\cos [2 \pi r x-(q+1) \pi / 2]}{(2 \pi r)^{1+q}}, \\
& q \geqq 0, \text { all } x ; 0>q>-1, \quad x \neq \text { integer. }
\end{aligned}
$$

For values of $x$ on the interval $(0,1)$, this coincides with $\zeta(-q, x)$, while, for integer $n$, we have

$$
\begin{aligned}
\bar{\zeta}(-q, n) & =\frac{1}{2}(\zeta(-q, 0)+\zeta(-q, 1)), & & q>0, \quad n=\text { integer } \\
\bar{\zeta}(0, n) & =0, & & n=\text { integer } .
\end{aligned}
$$

Carrying out this substitution, we find the

Euler-Maclaurin Asymptotic Expansion.

$$
\begin{aligned}
R^{[m, \nu]} f-I f= & \sum_{a=0}^{p_{b}-1} \frac{h^{(a)}(b)(-1)^{a} \bar{\zeta}\left(-(q+\beta), m b-t_{v}\right)}{q ! m^{q+\beta+1}} \\
& +\sum_{a=0}^{p_{a}-1} \frac{g^{(a)}(a) \bar{\zeta}\left(-(q+\alpha), t_{\nu}-m a\right)}{q ! m^{q+\alpha+1}}+E_{p_{b}, p_{a}}^{(m, \nu)} f
\end{aligned}
$$

where the remainder term is given by

$$
E_{p_{b}, p_{a}}^{(m, \nu)} f=2 \sum_{r=1}^{\infty} \operatorname{Re} e^{-2 \pi i r t,}\left(C_{p_{b}, p_{a}}^{(m r)} f+i S_{p_{b}, p_{a}}^{(m r)} f\right) .
$$

In view of order relation (2.2), it follows that

$$
E_{p_{b}, p_{a}}^{[m, v]} f \sim \mathrm{O}\left(m^{-\min \left(p_{b}+\beta+1, p_{a}+\alpha+1\right)}\right),
$$

and also we note that Restriction 2.5 is in force here. This is the variant of the EulerMaclaurin summation formula, valid for the function defined by (1.5). The subsidiary functions appearing on the right-hand side are defined by (1.10), (1.11) and (2.6). In addition (for $\alpha$ or $\beta$ negative), Restriction 2.5 is also applicable. This result has been previously published in Lyness and Ninham [5] in a different notation. 
3. The Singular Case. Before proceeding, it is convenient to examine carefully the nature of Restriction 2.5. This denies validity to the Euler-Maclaurin expansion (2.8) in the case when a function value $f(a)$ (or $f(b)$ ) is required for the rule sum and $\alpha\left(\right.$ or $\beta$ ) is negative. In this case, the rule sum $R^{[m, \nu]} f$ is undefined since one of its constituents $f(a)$ (or $f(b))$ is undefined.

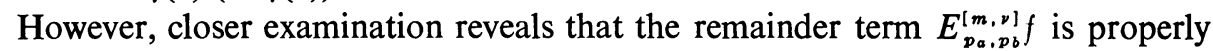
defined by (2.9) independently of this restriction. What happens in (2.8) is that an 'infinite' function value in the rule sum is precisely counterbalanced by an 'infinite' value of a generalized zeta function in the first coefficient in the expansion.

Subsequently, we shall wish to evaluate sums over index $m$ of expressions containing $E_{p_{a}, p_{b}}^{\left[m, p_{b}\right.} f$. In order to do this when $\alpha$ or $\beta$ is negative, we derive an alternate form of the Euler-Maclaurin expansion (2.8) which does not carry this inconvenient and inessential restriction. The result (Theorem 3.15 below) is simply that the 'infinite' function value may be ignored and that the 'infinite' coefficient is redefined in a specified and natural manner.

We now define a modified trapezoidal rule sum $R^{[m, \nu]} f^{*}$ as a version of $R^{[m, \nu]} f$ which 'ignores any singularity.' Specifically,

Definition 3.1.

$$
R^{[m, v]} f^{*}=\frac{1}{m} \sum_{j=1}^{m} f^{*}\left(\frac{j+t_{\nu}-1}{m}\right),
$$

where

$$
\begin{aligned}
f^{*}(x) & =f(x), & & \text { when this is defined, } \\
& =0, & & \text { otherwise. }
\end{aligned}
$$

Let us suppose that there is a singularity at $x=a$, that is $\alpha<0$. We recall that

$$
f(x)=(x-a)^{\alpha} g(x), \quad a<x<b .
$$

We define a function $f_{a}(x)$ which coincides with $f(x)$ in the vicinity of $x=a$ but is zero elsewhere as follows:

$$
\begin{array}{ll}
f_{a}(x)=(x-a)^{\alpha} g(x), & a<x<a+m / 2, \\
f_{a}(x)=0, & \text { elsewhere. }
\end{array}
$$

The function

$$
f_{s}(x)=f(x)-f_{a}(x)
$$

then coincides with $f(x)$ for $x>a+m / 2$ and is zero for $x<a+m / 2$.

We now take a value of $\nu$ for which $R^{[m, \nu]} f$ is defined, write down the EulerMaclaurin expansion (2.8) and subtract $R^{[m, \nu]} f_{a}$ from both sides. This gives

$$
\begin{aligned}
{\left[R^{[m, \nu]} f-R^{[m, \nu]} f_{a}\right]-I f=} & \sum_{a=0}^{p_{b}-1} \frac{h^{(q)}(b)(-1)^{a} \bar{\zeta}\left(-(q+\beta), m b-t_{\nu}\right)}{q ! m^{q+\beta+1}} \\
& +\sum_{a=1}^{p_{a}-1} \frac{g^{(a)}(a) \bar{\zeta}\left(-(q+\alpha), t_{\nu}-m a\right)}{q ! m^{q+\alpha+1}} \\
& +\left[g(a) \frac{\left.\overline{\zeta(}-\alpha, t_{\nu}-m a\right)}{m^{\alpha+1}}-R^{[m, \nu]} f_{a}\right]+E_{p_{b}, p_{a}}^{[m, \nu]}
\end{aligned}
$$


This formula, like the Euler-Maclaurin expansion, is valid only when $t_{v}-m a$ is not an integer. We now proceed to the limit as $t_{v}-m a$ approaches an integer. We denote this integer by $-j_{a}-1$, since then in the limit

$$
f\left(\frac{j_{a}+t_{\nu}-1}{m}\right)=f(a)
$$

and $j_{a}$ is the value of the index $j$ in the rule sum (1.9) at which the singularity is encountered. Consequently, we set $\nu$ to be a function of $\epsilon$ given by

$$
t_{\nu}-m a=-j_{a}-1+\epsilon
$$

and take the limit as $\epsilon \rightarrow 0$. The only terms in (3.5) in which a formal limiting process is required are those enclosed in square brackets. The first of these is evaluated as follows:

$$
\operatorname{Lim}_{\epsilon \rightarrow 0}\left[R^{[m, \nu]} f-R^{[m, \nu]} f_{a}\right]=\operatorname{Lim}_{\epsilon \rightarrow 0} R^{[m, \nu]} f_{s}=R^{[m, \nu]} f^{*} .
$$

This follows from (3.4) as $f_{s}(a)=0$ and (when $\left.|\epsilon|<m / 2\right) f_{s}\left(x_{i}\right)=f\left(x_{i}\right)$ for other values $x_{i}$ required by the rule sum.

We now treat the other term in square brackets. This is

$$
T(\epsilon)=g(a) \frac{\bar{\xi}\left(-\alpha, t_{\nu}-m a\right)}{m^{\alpha+1}}-R^{[m, \nu]} f_{a} .
$$

First, we note from (3.4) that, for $0<|\epsilon|<m / 2$,

$$
\begin{aligned}
R^{[m, \nu]} f_{a} & =\frac{1}{m} f_{a}\left(a+\frac{\epsilon}{m}\right)=\frac{1}{m}\left(\frac{\epsilon}{m}\right)^{\alpha} g\left(a+\frac{\epsilon}{m}\right), & 0 & <\epsilon<m / 2, \\
R^{[m, \nu]} f_{a} & =0, & -m / 2 & <\epsilon<0 .
\end{aligned}
$$

Thus, we treat separately the cases in which $\epsilon \rightarrow 0+$ and $\epsilon \rightarrow 0-$. In the second case, we find

$$
\begin{aligned}
\operatorname{Lim}_{\epsilon \rightarrow 0-} T(\epsilon) & =\frac{g(a)}{m^{\alpha+1}} \operatorname{Lim}_{\epsilon \rightarrow 0-} \bar{\zeta}\left(-\alpha,-j_{a}-1+\epsilon\right) \\
& =\frac{g(a)}{m^{\alpha+1}} \operatorname{Lim}_{\epsilon \rightarrow 0-} \zeta(-\alpha, 1+\epsilon)=g(a) \zeta(-\alpha, 1) / m^{\alpha+1}
\end{aligned}
$$

The first case is more complicated. For $0<\epsilon<m / 2$, we may use (3.10) to express (3.9) in the form

$$
T(\epsilon)=\frac{g(a)}{m^{\alpha+1}}\left(\zeta(-\alpha, \epsilon)-\epsilon^{\alpha}\right)-\frac{g(a+\epsilon / m)-g(a)}{\epsilon / m} \frac{1}{m}\left(\frac{\epsilon}{m}\right)^{\alpha+1} .
$$

Since $g^{\prime}(a)$ exists and $\alpha+1>0$, the second expression in (3.12) has the limiting value zero. Using the identity

$$
\zeta(-\alpha, x)=x^{\alpha}+\zeta(-\alpha, x+1),
$$

it follows that

$$
\operatorname{Lim}_{\epsilon \rightarrow 0+} T(\epsilon)=g(a) \zeta(-\alpha, 1) / m^{\alpha+1}
$$


which coincides with (3.11).

If there is a singularity at $b$, i.e. $\beta<0$, a similar derivation leads to a corresponding result. Consequently, we are able to state what adjustments are necessary to the Euler-Maclaurin expansion (2.8) in order to take into account ignoring a singularity. These are as follows:

THEOREM 3.15. Expansion (2.8) is valid, with $R^{[m, \nu]} f^{*}$ defined in (3.1) replacing $R^{[m, \nu]}$ so long as coefficients $\bar{\zeta}(-\beta, 1)$ and $\bar{\zeta}(-\alpha, 1)$ when they occur with $\beta<0$ and $\alpha<1$ are replaced by $\zeta(-\beta, 1)$ and $\zeta(-\alpha, 1)$, respectively.

Though we do not require it here, Theorem 3.15 is the nontrivial part of a more general result, valid for all values of $\alpha, \beta>-1$. Thus,

THEOREM 3.16. In cases where $R^{[m, \nu]}$ finvolves a function value at $x=a$, this function value may be ignored in the Euler-Maclaurin expansion (2.8) if at the same time the term $\bar{\zeta}(-\alpha, 1)$ is replaced by $\zeta(-\alpha, 1)$.

Proof. When $\alpha<0$, this follows from Theorem 3.15.

When $\alpha>0$, the function value is zero and $\bar{\zeta}(-\alpha, 1)=\zeta(-\alpha, 1)$.

When $\alpha=0$, a term $g(a) / 2 m$ is omitted from the rule sum and in the expansion the term $g(a) \bar{\zeta}(0,1) / m$ is replaced by $g(a) \zeta(0,1) / m$. Since $\zeta(0,1)-\bar{\zeta}(0,1)=\frac{1}{2}$, these two adjustments balance each other.

One may go on from Theorem 3.15 to construct a quantitative theory of ignoring the singularity, valid only for this special type of singularity. However, the interest in this paper is that the term $E_{p_{b}, p_{a}}^{[m, p]}$ generally defined by (2.8) may be evaluated even if (2.8) breaks down. All that is necessary is that (2.8) be reinterpreted in the context of ignoring the singularity and adjusting a single term in conformity with Theorem 3.15.

4. Möbius Inversion. We may now proceed to the Möbius inversion of specific sets of equations obtained from (2.9), which relate the remainder terms in the different expansions. There are many possibilities. The simplest is to set $\nu=1$ in (2.9) giving

$$
E_{p_{b}, p_{a}}^{(m, 1)} f=2 \sum_{r=1}^{\infty} C_{p_{b}, p_{a}}^{(m r)} f .
$$

The order of the terms on the right for large $r$ is given by

$$
C_{p_{b}, p_{a}}^{(m r)} \sim O\left(r^{-\min \left(p_{b}+\beta+1, p_{a}+\alpha+1\right)}\right) .
$$

To apply the Möbius inversion technique, it is therefore sufficient to require that

$$
p_{b}+\beta>0 ; \quad p_{a}+\alpha>0
$$

and the result is

$$
2 C_{p_{b}, p_{a}}^{(m)} f=\sum_{s=1}^{\infty} \mu_{s} E_{p_{b}, p_{a}}^{[m s, 1]} f,
$$

where $\mu_{s}$ is the sth Möbius number defined by

$$
\begin{aligned}
& \mu_{1}=1, \\
& \mu_{j}=0 \\
& \mu_{i}=(-1)^{r} \quad \text { if } j \text { has a square factor other than 1, }
\end{aligned}
$$


Other equations may be derived from (2.9) in a similar way. For example,

$$
\begin{aligned}
2 C_{p_{b}, p_{a}}^{(m)} f & =-\sum \nu_{s} E_{p_{b}, p_{a}}^{[m s, 0]} f \\
& =-\sum \bar{\nu}_{s} \frac{1}{2}\left(E_{p_{b}, p_{a}}^{[m s, 0]} f-E_{p_{b}, p_{a}}^{[2 m s, 0]} f\right) \\
& =-\sum \bar{\nu}_{s}^{* 1} \frac{1}{2}\left(E_{p_{b}, p_{a}}^{[m s, 0]} f-E_{p_{b}, p_{a}}^{[3 m s, 0]} f\right) \\
& =\sum_{s=1}^{\infty} \mu_{2 s-1}\left(E_{p_{b}, p_{a}}^{[m(2 s-1), 1]} f-E_{p_{b}, p_{a}}^{[2 m(2 s-1), 1]} f\right) \\
2 S_{p_{b}, p_{a}}^{(m)} f & =\sum_{s=1}^{\infty}(-1)^{s-1} \mu_{2 s-1} \frac{1}{2}\left(E_{p_{b}, p_{a}}^{[m(2 s-1),-1 / 2]} f-E_{p_{b}, p_{a}}^{[m(2 s-1),+1 / 2]} f\right) .
\end{aligned}
$$

The coefficients are given by

$$
\begin{array}{rlrl}
\bar{\nu}_{i} & =\nu_{i}=\mu_{i}, & & j \text { odd }, \\
\bar{\nu}_{i}=2 \nu_{i}=2^{n} \mu_{k}, & & j=2^{n} k ; k \text { odd, } \\
\bar{\nu}_{i}^{*}=\nu_{i}, & j=3 k \pm 1, \\
\bar{\nu}_{i}^{*}=0, & j=3 k .
\end{array}
$$

The various Eqs. (4.4) to (4.8) have slightly different characteristics. The first two require the value of If which, if available, leads to a shorter calculation. The second, third and fourth do not require function values at $x=0$ and $x=1$. This is convenient when the interval is $[0,1]$ and $\alpha$ or $\beta$ are negative. (4.7) would not normally be used computationally, and is mentioned only because it is a natural correspondent to (4.9).

We write out in full Eq. (4.4). This is:

$$
\begin{aligned}
2 C^{(m)} f= & 2 \sum_{a=0}^{p_{b}-1} \frac{h^{(a)}(b)(-1)^{q}(q+\beta) !}{q !(2 \pi m)^{q+\beta+1}} \cos [2 \pi m b-\pi(q+\beta+1) / 2] \\
& +2 \sum_{q=0}^{p_{a}-1} \frac{g^{(a)}(a)(q+\alpha) !}{q !(2 \pi m)^{q+\alpha+1}} \cos [2 \pi m a+\pi(q+\alpha+1) / 2] \\
& +\sum_{s=1}^{\infty} \mu_{s}\left[R^{[m s, 1]} f-I f-\sum_{q=0}^{p_{b}-1} \frac{h^{(a)}(b)(-1)^{q} \bar{\zeta}(-(q+\beta), m s b)}{q !(m s)^{q+\beta+1}}\right. \\
& \left.-\sum_{q=0}^{p_{a}-1} \frac{g^{(a)}(a) \bar{\zeta}(-(q+\alpha),-m s a)}{q !(m s)^{q+\alpha+1}}\right] .
\end{aligned}
$$

The other Eqs. (4.5)-(4.8) have a similar structure, the difference being that the terms in the sum over index $s$ are different. As is the case in Parts I and II, these equations are identities in the coefficients $h^{(a)}(b)$ and $g^{(a)}(a), q=1,2, \cdots$, and they are also identities in $h(b)$ (or $g(a)$ ) if $\alpha$ (or $\beta$ ) is positive. To establish this, we write definition (2.6) of $\bar{\zeta}(-q, x)$ in the form

$$
\frac{\bar{\zeta}(-q, m x)}{m^{1+q}}=2 q ! \sum_{r=1}^{\infty} \frac{\cos [2 \pi r m x-(q+1) \pi / 2]}{(2 \pi m r)^{1+q}} .
$$

Since this may be expressed as

$$
G(m)=\sum_{r=1}^{\infty} F(r m),
$$


where

$$
\begin{aligned}
& G(m)=\bar{\zeta}(-q, m x) / m^{1+q}, \\
& F(m)=2 q ! \cos (2 \pi m x-(q+1) \pi / 2) /(2 \pi m)^{1+q},
\end{aligned}
$$

it may be formally inverted as follows:

$$
F(m)=\sum_{s=1}^{\infty} \mu_{s} G(s m)
$$

Thus,

$$
2 q ! \frac{\cos (2 \pi m x-(q+1) \pi / 2)}{(2 \pi m)^{1+q}}=\sum_{s=1}^{\infty} \frac{\mu_{s} \bar{\zeta}(-q, m s x)}{(m s)^{1+q}} .
$$

This inversion is validated using the Möbius inversion theorem $(\mathrm{I}, 5.4)$ when $q>0$, since then

$$
\sum_{l=1}^{\infty} \sum_{k=1}^{\infty}\left|\mu_{l} F(k l m)\right|<\frac{2 q !}{(2 \pi)^{1+q}}(\zeta(1+q))^{2}<\infty .
$$

Replacing $x$ by $b$ and $q$ by $q+\beta$ in (4.16), we find

$$
\begin{aligned}
2(q+\beta) ! \frac{\cos (2 \pi m b-(q+\beta+1) \pi / 2)}{(2 \pi m)^{q+\beta+1}}-\sum_{s=1}^{\infty} \frac{\mu_{s} \bar{\zeta}(-(q+\beta), m s b)}{(m s)^{1+q}} & =0, \\
q+\beta & >0 .
\end{aligned}
$$

In other words, the coefficient of $h^{(a)}(b) / q$ ! in Eq. (4.11) is precisely zero.

Thus, Eq. (4.11) is an identity in $h^{(a)}(b)$ and in $g^{(a)}(a)$ when $q+\beta>0$ and $q+\alpha$ $>0$ and it remains valid if these numbers are arbitrarily replaced by other numbers. In particular, $h^{(a)}(b)$ and $g^{(a)}(a)$ may be replaced by approximate values $\tilde{h}^{(a)}(b)$ and $\tilde{g}^{(a)}(a)$.

The effect of using inaccurate derivatives in the MIPS calculation has been described in Section 9 of Part I. This is to increase the number of terms $E_{p_{b}, p_{a}}^{[s, 1]} f$ which have to be calculated and so to lengthen the calculation. However, the overall accuracy is not impaired.

5. Comments on the Calculation. It is conventional, before publishing formulas for use in numerical computation, for the author to satisfy himself that the formulas are valid or useful by carrying out some test problems. In this instance, a FORTRAN coded program has been written. This implements Eq. (4.11) and evaluates approximations $2 \tilde{C}^{(m)} f$ to

$$
2 C^{(m)} f=2 \int_{a}^{b}(x-a)^{\alpha}(b-x)^{\beta} \phi(x) \cos 2 \pi m x d x, \quad m=1,2, \cdots,
$$

each approximation having alleged absolute accuracy $\epsilon$. The code naturally requires as input parameters the values of $\epsilon, a, b, \alpha, \beta$ and If. In addition, it requires a subroutine for $\phi(x)$ for all $x \in[a, b]$ and a subroutine to provide the derivatives $\phi^{(a)}(a)$, $\phi^{(a)}(b), q=0,1,2, \cdots, q_{\max }$. The routine returns two sets of coefficients

$$
\begin{aligned}
& \bar{p}\left(\leqq q_{\max }\right), A_{1}, B_{1}, A_{2}, B_{2}, \cdots, A_{\bar{p}}, B_{\bar{p}}, \\
& \bar{s}(\leqq 100), 2 C^{(m)} f, \quad(m=1,2, \cdots, \bar{s}) .
\end{aligned}
$$


These may be used in the formula

$$
\begin{aligned}
2 \tilde{C}^{(m)} f= & 2 \sum_{q=1}^{\bar{p}} B_{q} \cos [2 \pi m b-\pi(q+\beta) / 2] / m^{a+\beta} \\
& +2 \sum_{q=1}^{\bar{p}} A_{q} \cos [2 \pi m a+\pi(q+\alpha) / 2] / m^{q+\alpha}, \quad m>\bar{s},
\end{aligned}
$$

to evaluate whichever of the Fourier coefficients are required. The approximation error is supposed to satisfy

$$
2\left|\tilde{C}^{(m)} f-C^{(m)} f\right|<\epsilon .
$$

(For brevity, various details and other options have been omitted from this description.)

This program has been checked quite thoroughly in the cases in which $\alpha=\beta=0$ as in that case there are many functions $\phi(x)$ for which a simple expression for $C^{(m)} f$ is known. This is the case dealt with in Parts I and II.

The testing of the routine for noninteger $\alpha, \beta$, and particularly for $\alpha, \beta$ negative, was of particular interest. The main difficulty is that there appear to be no readily available special cases in which a simple expression for $C^{(m)} f$ exists and no other methods designed for this problem. Thus, in general, one had to resort to numerical integration using an automatic quadrature routine. This is very time consuming. One needs to carry out a series of different integrations for different values of $m$. Each involves an integrand having two infinite singularities and which is oscillatory. The results were nearly always consistent. In the cases in which they were not, the error was traced to the automatic quadrature routine.

It is, of course, quite unrealistic to expect a general automatic quadrature routine to be nearly as efficient as a carefully constructed special method. In general, it was more time consuming to carry out the easiest of these integrations, i.e. to evaluate the single Fourier coefficient $C^{(1)} f$ than to calculate the entire set using the MIPS method. These time factors varied between 20 and 2000 .

Because of the large amount of machine time required, the testing was not as thorough as is usually the case in testing quadrature methods.

One special case was tested relatively thoroughly. This was the case $\alpha=\beta=-\frac{1}{2}$. Here a simple substitution may be used to express the integrand in a form without singularities. The numerical quadrature was then much faster. In this case, results were consistently accurate and no discrepancies were noted. And the time factor mentioned above became about 1 . That is to say that if only $C^{(m)} f, m=1$ or 2 were required, it was roughly equally time consuming to use the MIPS method as to make the transformation analytically and to use an automatic routine.

At present, the only tests carried out are those described above. The following example provides a rough indication of the magnitudes involved in the MIPS calculation.

The routine was used with

$$
a=0.2, \quad b=0.9, \quad \alpha=-0.4, \quad \beta=-0.7, \quad \phi(x)=1, \quad \epsilon=10^{-8} .
$$

The early cosine Fourier coefficients are of roughly unit magnitude and $2 C^{(1000)} f=$ 0.46. Ultimately, 


$$
C^{(m)} f \sim O\left(m^{-0.3}\right) \text { as } m \rightarrow \infty .
$$

The routine found $\bar{s}=15$. (At this stage four members of the sequence $E_{5,5}^{[s, 1]} f$ were smaller in magnitude than $10^{-8}$.)

Thus, on the basis of exact derivatives $\phi^{(a)}(a), \phi^{(a)}(b), q=0,1,2,3,4$, and about 50 function evaluations of $f(x)$, the routine provided the values of the first fifteen Fourier coefficients and a set of ten numbers, which, used in (5.3), provided approximations of alleged accuracy $10^{-8}$ to any of the Fourier coefficients.

Argonne National Laboratory

9700 South Cass Avenue

Argonne, Illinois 60439

1. J. N. LYNESS, "The calculation of Fourier coefficients by the Möbius inversion of the Poisson summation formula. Part I. Functions whose early derivatives are continuous," Math. Comp., v. 24, 1970, pp. 101-135.

2. J. N. LYNESS, "The calculation of Fourier coefficients by the Möbius inversion of the Poisson summation formula. Part II. Piecewise continuous function and functions with poles near the interval $[0,1]$," Math. Comp., v. 25, 1971, pp. 59-78.

3. A. ERDÉLYI, "Asymptotic representations of Fourier integrals and the method of stationary phase," J. Soc. Indust. Appl. Math., v. 3, 1955, pp. 17-27. MR 17, 29.

4. J. N. LyNESS, "Adjusted forms of the Fourier coefficient asymptotic expansion and applications in numerical quadrature," Math. Comp., v. 25, 1971, pp. 87-104.

5. J. N. LYNESS \& B. W. NINHAM, "Numerical quadrature and asymptotic expansions," Math. Comp., v. 21, 1967, pp. 162-178. MR 37 \#1081. 